\title{
Severe Autoinflammatory Manifestations and Antibody Deficiency Due to Novel Hypermorphic PLCG2 Mutations
}

\author{
Andrea Martín-Nalda ${ }^{1,2} \cdot$ Claudia Fortuny ${ }^{3,4,1} \cdot$ Lourdes Rey $^{5} \cdot$ Tom D. Bunney ${ }^{6} \cdot$ Laia Alsina $^{4,7,8} \cdot$ Ana Esteve-Solé ${ }^{4,7,8}$. \\ Daniel Bull ${ }^{9}$. Maria Carmen Anton ${ }^{10}$ - María Basagaña ${ }^{11}$ • Ferran Casals $^{12}$ - Angela Deyá ${ }^{4,7,8} \cdot$ Marina García-Prat $^{1,2}$. \\ Ramon Gimeno ${ }^{13}$. Manel Juan ${ }^{10,14,15} \cdot$ Helios Martinez-Banaclocha ${ }^{16}$. Juan J Martinez-Garcia ${ }^{16}$. \\ Anna Mensa-Vilaró ${ }^{10}$. Raquel Rabionet ${ }^{4,17}$. Nieves Martin-Begue ${ }^{18}$. Francesc Rudilla ${ }^{19,20}$. Jordi Yagüe ${ }^{10,14,15}$. \\ Xavier Estivill ${ }^{21}$. Vicente García-Patos ${ }^{22}$ • Ramon M. Pujol ${ }^{23}$. Pere Soler-Palacín 1,2,24 Matilda Katan $^{6}$. \\ Pablo Pelegrín ${ }^{16} \cdot$ Roger Colobran ${ }^{2,25,26} \cdot$ Asun Vicente $^{27}$ • Juan I. Arostegui ${ }^{10,14,15}$
}

Received: 10 January 2020 / Accepted: 20 May 2020 / Published online: 15 July 2020

(C) The Author(s) 2020

\begin{abstract}
Autoinflammatory diseases (AIDs) were first described as clinical disorders characterized by recurrent episodes of seemingly unprovoked sterile inflammation. In the past few years, the identification of novel AIDs expanded their phenotypes toward more complex clinical pictures associating vasculopathy, autoimmunity, or immunodeficiency. Herein, we describe two unrelated patients suffering since the neonatal period from a complex disease mainly characterized by severe sterile inflammation, recurrent bacterial infections, and marked humoral immunodeficiency. Whole-exome sequencing detected a novel, de novo heterozygous PLCG2 variant in each patient (p.Ala708Pro and p.Leu845_Leu848del). A clear enhanced PLC 22 activity for both variants was demonstrated by both ex vivo calcium responses of the patient's B cells to IgM stimulation and in vitro assessment of PLC activity. These data supported the autoinflammation and PLC $\gamma 2$-associated antibody deficiency and immune dysregulation (APLAID) diagnosis in both patients. Immunological evaluation revealed a severe decrease of immunoglobulins and B cells, especially class-switched memory B cells, with normal T and NK cell counts. Analysis of bone marrow of one patient revealed a reduced immature B cell fraction compared with controls. Additional investigations showed that both PLCG2 variants activate the NLRP3-inflammasome through the alternative pathway instead of the canonical pathway. Collectively, the evidences here shown expand APLAID diversity toward more severe phenotypes than previously reported including dominantly inherited agammaglobulinemia, add novel data about its genetic basis, and implicate the alternative NLRP3-inflammasome activation pathway in the basis of sterile inflammation.
\end{abstract}

Keywords Autoinflammatory diseases · APLAID · PLC $\gamma 2 \cdot$ inflammasome $\cdot$ caspase- $1 \cdot$ interleukin- 1 - agammaglobulinemia

\section{Introduction}

Andrea Martin-Nalda and Claudia Fortuny are first co-authors who contributed equally to this article.

Pablo Pelegrín, Roger Colobran, Asun Vicente, and Juan I. Arostegui contributed equally to this article as senior authors.

Electronic supplementary material The online version of this article (https://doi.org/10.1007/s10875-020-00794-7) contains supplementary material, which is available to authorized users.

Juan I. Arostegui

jiaroste@clinic.cat

Extended author information available on the last page of the article
Autoinflammatory diseases (AIDs) constitute a specific group of primary immunodeficiency diseases (PID) characterized by recurrent episodes of systemic sterile inflammation mainly mediated by cells of innate immunity [1]. At present, around 30 different monogenic AIDs have been molecularly elucidated, with some of the novel ones also displaying features of dysregulated B cell response either as circulating autoantibodies or as reduced antibody production [2-5]. The extremely rare, dominantly inherited PLC $\gamma 2$-associated antibody deficiency and immune dysregulation (PLAID) and autoinflammation and PLC $\gamma 2$ associated antibody deficiency and immune dysregulation (APLAID) may be included among those rare monogenic AIDs combining sterile inflammation and humoral 
immunodeficiency. Both diseases are linked to hypermorphic, pathogenic variants in the PLCG2 gene encoding the key signal transduction enzyme PLC $\gamma 2$. They are clinically characterized by early-onset skin inflammation and recurrent infections. In addition, patients with APLAID often develop ocular and lung inflammation, enterocolitis, and interstitial pneumonitis but unlike PLAID, they do not present with cold urticaria or autoimmunity [6-9]. However, because only a few APLAID cases have been characterized so far, it is not clear to what extent their clinical manifestations could differ or overlap with other immune disorders. Similarly, the range of genetic changes (in PLCG2 or in genes regulating associated signal transduction pathways) and their impact on immune cell functions have not been defined.

In the present study we describe two unrelated patients with early-onset severe skin and eye inflammation, and recurrent bacterial infections secondary to antibody deficiency. Further genetic and functional studies are consistent with APLAID and consolidate and expand the key features and underpinning molecular mechanisms for this diagnosis.

\section{Patients and Methods}

The ethics committees of Hospital Sant Joan de Déu, Hospital Universitari Vall d'Hebron and Hospital Clínic, all in Barcelona (Spain), approved the study. Written informed consent for participation in the study was obtained from patients' parents. Blood samples from patients and unaffected relatives were collected for molecular studies, which were performed in accordance with the Declaration of Helsinki.

\section{Flow Cytometry Studies}

Peripheral blood mononuclear cells (PBMCs) were isolated by Ficoll gradient (Fresenius-Kabi Norge, Norway) and stained for cell surface markers using fluorochrome-conjugated antibodies (Supplementary Table S1). Samples were acquired using a FACSCanto II cytometer (BD Biosciences, USA), and data were analyzed with CellQuest software (BD Biosciences, USA).

\section{Genetic Analyses}

DNA samples were extracted from peripheral blood using a QIAmp DNA Blood Mini Kit (Qiagen, Germany). Libraries were prepared using TruSight One kit (Illumina, USA) in Family 1 and SureSelect Human All exon V2 kit (Agilent Technologies, USA) in Family 2 following manufacturer's instructions. Paired-end sequencing was performed on an Illumina Genome Analyzer II platform (Illumina, USA). Reads were mapped against the human reference genome hg19 using the BWA software and analyzed with the GATK Unified Genotyper v2.8. Amplicon-based deep sequencing of specific exons of the PLCG2 gene (RefSeq NM_002661.3) was performed as previously described to evaluate parental gene mosaicism [10]. For Sanger sequencing, specific exons of the PLCG2 gene were amplified by in house-designed PCR (primers listed in Supplementary Table S2), purified with Illustra ExoStar 1-Step kit (GE Healthcare, USA), bidirectional sequenced using ABI BigDye ${ }^{\circ}$ Terminator v3.1 Cycle Sequencing Kit (Applied Biosystems, USA) and run on an automated ABI 3730XL analyzer (Applied Biosystems, USA).

\section{Analyses of $\mathrm{Ca}^{2+}$ Flux in B Cells and Measurements of Phosphoslipase-C Activity}

Intracellular $\mathrm{Ca}^{2+}$ flux was measured by flow cytometry after labeling with FLUO-3 AM (Invitrogen, USA) as previously described with slight modifications [6].

For the measurements of phosphoslipase-C (PLC) activity, COS-7 cells were cultured in DMEM (Sigma-Aldrich, USA) containing $10 \%$ (v/v) FBS and $2.5 \mathrm{mM}$ glutamine (growth media). Cells were grown as a monolayer at $37{ }^{\circ} \mathrm{C}$ in $5 \% \mathrm{CO}_{2}$. COS-7 cells were seeded into 96-well plates at a density of 7500 cells/well in $0.1 \mathrm{~mL}$ of growth media and incubated overnight. Fresh media was applied and the cells transfected with plasmid DNA at $100 \mathrm{ng} /$ well that had been diluted in $5 \mu \mathrm{L}$ jetPRIME® buffer and $0.2 \mu \mathrm{L}$ jetPRIME® (Polyplus Transfection, France) that was prepared as instructed by the manufacturer. The DNA concentration was kept constant by adding empty plasmid. For the PLC $\gamma 2$ expression plasmids, the full-length ORF of human PLCG2 was cloned into the vector pTriEx4 using Gateway technology (Thermo Fisher). Mutations and deletions were prepared using the site-directed mutagenesis kit (Agilent) following manufacturer's instructions. The ORFs of all constructs were fully sequenced prior to a Maxiprep being performed to generate the plasmid DNA for transfection. Each PLC $\gamma 2$ construct was transfected at 4 concentrations in triplicate as outlined in the figures. Twenty-four hours post-transfection, the media was removed and replaced with growth media without FBS but containing $0.25 \%(\mathrm{w} / \mathrm{v}$ ) fatty acid free BSA. The COS-7 cells were then incubated for further $24 \mathrm{~h}$. Subsequently, the media was replaced with growth media without FBS but containing $50 \mathrm{mM} \mathrm{LiCl}$ with and without $100 \mathrm{ng} / \mathrm{mL}$ EGF and incubated for further $1 \mathrm{~h}$. The media was aspirated and replaced by $25 \mu \mathrm{L}$ of stimulation buffer ( $20 \mathrm{mM}$ HEPES.OH, $2 \mathrm{mM} \mathrm{CaCl}_{2}, 1 \mathrm{mM} \mathrm{MgCl}, 8.4 \mathrm{mM} \mathrm{KCl}, 292 \mathrm{mM} \mathrm{NaCl}$, $11 \mathrm{mM}$ glucose and $100 \mathrm{mM} \mathrm{LiCl}, \mathrm{pH}$ 7.4) followed by $25 \mu \mathrm{L}$ of lysis buffer (50 mM HEPES.OH, $0.8 \mathrm{M} \mathrm{KF}, 0.2 \%$ (w/v) BSA and $1 \%(\mathrm{v} / \mathrm{v})$ Triton-X-100, $\mathrm{pH}$ 7.0). The cells were lysed for $30 \mathrm{~min}$ at room temperature on an orbital shaker. Seven microliters of the cell lysate was pipetted in duplicate into a white 384well plate (Greiner Bio-One $\mathrm{GmbH}$, Austria) followed by $1.6 \mu \mathrm{L}$ of IP1-d2 (Cisbio, France). After 5 min, $1.6 \mu \mathrm{L}$ of anti-IP1Cryptate (Cisbio, France) was added and the plate sealed and incubated at room temperature for $1 \mathrm{~h}$. The plate was read on a PHERAstar (BMG Labtech, Germany) plate reader in HTRF mode, and the data converted to $\mathrm{IP}_{1}$ concentration using a standard 
curve generated following manufacturer's instructions. Data for the measured PLC activity represent the standard error of the mean of transfections performed in triplicate.

Quantities of expressed proteins were measured using a WES Western Blotting system (Protein Simple, USA). For this, a further 96-well plate was transfected identically to the plate used in the $\mathrm{IP}_{1}$ assay described above. Forty-eight hours post-transfection, the cells were washed once in ice-cold PBS and subsequently lysed in $20 \mu \mathrm{L}$ RIPA buffer (Thermofisher, USA) containing a protease and phosphatase inhibitor cocktail (Thermofisher, USA). The cells were freeze-thawed at $-20^{\circ} \mathrm{C}$ and subsequently $4 \mu \mathrm{L}$ of each well loaded on a WES Western Blotting. Proteins were detected with a 1:150 dilution of the antiPLC $\gamma 2$ antibody sc5283 (Santa Cruz) and a 1:150 dilution of the anti- $\beta$-actin antibody 13E5 (Cell Signaling Technology). For the comparison of PLC activity of different PLC $\gamma 2$ variants, the expression levels were quantitated and points with the same protein expression used, as previously described [11]. The differences were confirmed using the 2 tailed $t$ test.

\section{Detection of Intracellular ASC Specking and Active Caspase-1}

PBMCs were treated with either nothing or E. coli LPS serotype 055:B5 (Sigma-Aldrich, USA; $1 \mu \mathrm{g} / \mathrm{mL}, 2 \mathrm{~h}$ at $37^{\circ} \mathrm{C}$ ). LPSprimed PBMCs were then stimulated with either nothing, ATP $(3 \mathrm{mM})$, or nigericin $(10 \mu \mathrm{M})$ for $30 \mathrm{~min}$ at $37^{\circ} \mathrm{C}$. Stimulated PBMCs were fixed with $2 \%$ paraformaldehyde (Sigma-Aldrich, USA) and stained for the detection of intracellular ASC specks by TOFIE as previously described [12], using the rabbit polyclonal antibody anti-ASC (N-15)-R (Santa Cruz Biotechnology, USA).

For active caspase-1 detection, PBMCs were incubated for $1 \mathrm{~h}$ with FLICA660 reagent (ImmunoChemistry Technologies, USA) and fixed following manufacturer recommendations. Monocytes were detected with the APC-vio770-conjugated anti-human CD33 antibody (Miltenyi Biotec, Germany) and with the APC-Cy7-conjugated anti-human CD14 antibody (TONBO Biosciences, USA). Stained cells were acquired on a FACSCanto cytometer (BD Biosciences, USA). Cytokines were measured in cell supernatants using a custom bead-based multiplex Luminex immunoassay (eBioscience, USA). Heat maps representing cytokine expression profiles were created using Morpheus software (Broad Institute, Cambridge, USA).

\section{Results}

\section{Clinical Description}

\section{Family 1}

Patient 1 has been first described in 2007 [13] and is now a 16year-old girl. She was born from healthy parents at 40 weeks of gestation by cesarean section due to loss of fetal wellbeing (pedigree in Fig. 1a). The main features of her disease included early-onset severe cutaneous and eye inflammation and recurrent respiratory infections (Table 1).

Skin lesions appeared as early as the first day of life as numerous papulo-vesicular lesions, which became generalized during the following days requiring admission into pediatric intensive care unit. These lesions have been nearly continuously present, with exacerbations, occasionally hemorrhagic and complicated with infections, ulcerated lesions, and ulcerative granulomata (Fig. 2a). In recent years, large areas of cutis laxa and hyperpigmentation were detected (Fig. 2b). At 2.5 years of age, bilateral conjunctivitis, corneal erosions, and nodules appeared (Fig. 2c, d).

The absence of circulating immunoglobulins was detected during the first year of life (Table 2). Intravenous immunoglobulin (IVIG) replacement therapy (IVIGs; $400 \mathrm{mg} / \mathrm{kg} \mathrm{q} 3 \mathrm{w}$ ) was then started and has been maintained until present. Despite this treatment, multiple infections were detected (cutaneous infections, acute gastroenteritis, periodontitis, herpetic stomatitis, bronchitis, and pneumonia). At 4 years of age, multiple central bronchiectases were detected on a CT scan (Fig. 2e), which subsequently progressed and provoked recurrent episodes of acute hemoptysis that required urgent embolization. As consequence of these lesions, the medium right lung lobe was surgically excised at the age of 13 years.

The patient received multiple treatments including antibiotics, retinoids, corticosteroids, etanercept ( $25 \mathrm{mg}$ q1w for 5 years), and anakinra (100 mg q1d for 1.5 years). With the use of etanercept and anakinra, a partial control of skin inflammation was detected, with no improvement of ocular manifestations or immune defects. By contrast, a marked decrease of plasma C-reactive protein (CRP) was detected with etanercept (mean $6.86 \mathrm{mg} / \mathrm{L}$; range 0.08-21-8) and anakinra (mean $0.10 \mathrm{mg} / \mathrm{L}$; range 0.05-0.16) compared with periods in which these treatments were not administered (mean $13.32 \mathrm{mg} / \mathrm{L}$; range 1.3-31.63).

\section{Family 2}

Patient 2 is a 9-year-old boy born from healthy parents (pedigree in Fig. 1a). Skin manifestations started during the first week of life as multiple erythematous macules, papules, and large plaques, mainly located at arms, abdomen, and thighs (Table 1). These lesions recurred with no periodicity, sometimes presenting as urticaria-like, vesicular or pustular lesions (Fig. 2f) or ulcerated or exudative plaques. Infections, minor traumas, vaccinations, and heat were identified as triggering or worsening factors. They were successfully treated with oral or topical corticosteroids (1 $\mathrm{mg} / \mathrm{kg} \mathrm{q} 1 \mathrm{~d})$, and partially with dapsone $(1 \mathrm{mg} / \mathrm{kg}$ q1d), and they healed leaving focal, wrinkled-appearing 
a Family 1 Family 2

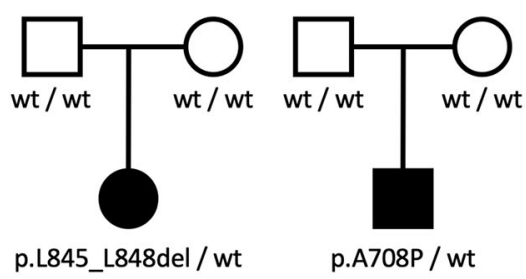

b

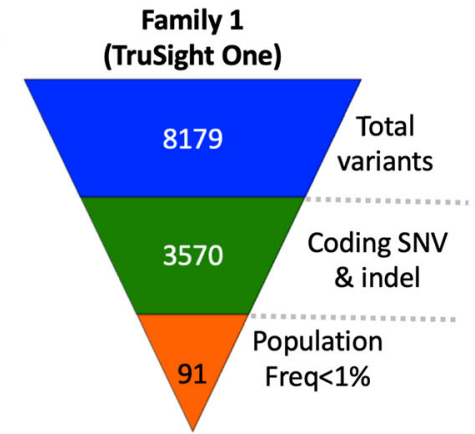

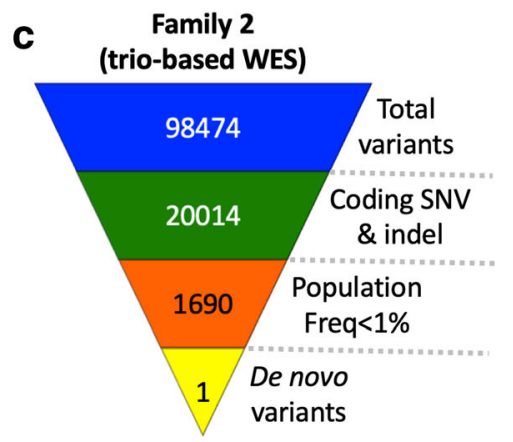

d Patient 1

c.2533_2544del TTAGGGTCTCTT p.L845_L848del

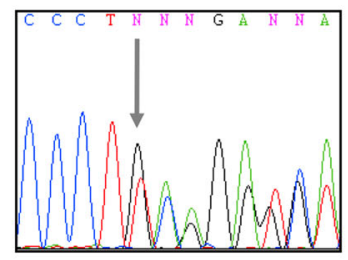

Control

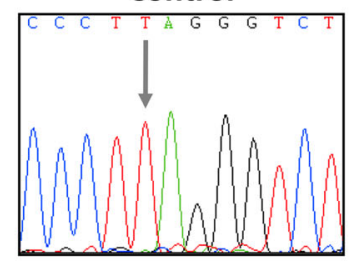

Patient 2 c. $2122 \mathrm{G}>\mathrm{C}$ p.A708P

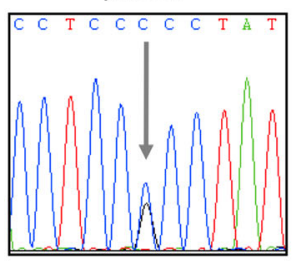

Control

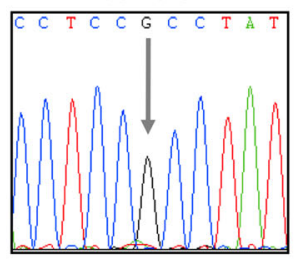

e

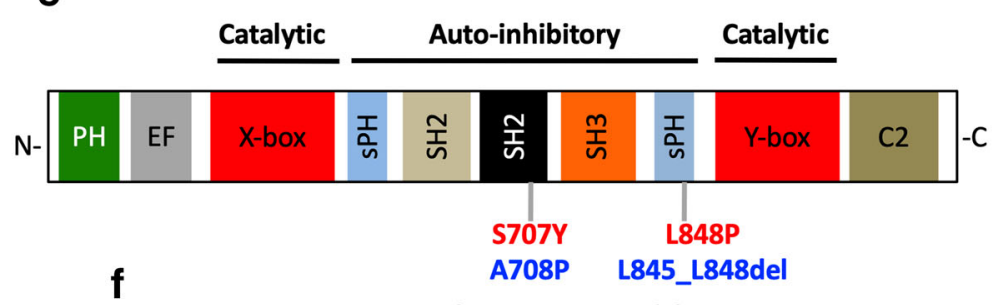

Fig. 1 Familial pedigrees and features of $P L C G 2$ variants. Panel a Pedigrees of enrolled families. Black filled symbols represent affected individuals, open symbols unaffected individuals, squares male individuals and circles female individuals. Panels b, c Schemes of filtering of the next-generation sequencing strategies used in the enrolled families. Panel d Sanger sense chromatograms of the PLCG2 gene from patients (upper boxes) and from wild-type healthy subjects (bottom boxes). Gray arrows indicate the PLCG2 variants detected in patches of cutis laxa and residual hyperpigmentation (Fig. $2 \mathrm{~g}$ ). Ocular inflammatory manifestations have recently appeared as bilateral red eye, corneal limbitis, and episcleritis (Fig. 2h).

Infections started at the age of 2 months as recurrent, mild viral bronchitis. Since the age of 4 years, recurrent bacterial infections were detected, mainly at ear (>10 episodes) and lung (4 pneumonias), leading to mild bronchiectasis (Fig. 2e). All infections were successfully treated with oral antibiotics, without hospitalization. IVIG replacement therapy $(500 \mathrm{mg} / \mathrm{kg} \mathrm{q} 3 \mathrm{w})$ was started in January 2016, which resulted in a decrease of the frequency of respiratory infections and in a marked improvement of patient's health status.

\section{Hematological and Immunological Parameters}

Laboratory monitoring revealed increased inflammatory markers (CRP, platelet count) and reduced hemoglobin in both patients (Supplementary Fig. S1). Immunological tests repeatedly revealed low-to-absent immunoglobulins and marked decrease of B cells in both patients, with $\mathrm{T}$ and NK cell counts repeatedly normal (Table 2). A comprehensive analysis of circulating B cells revealed their near complete absence in patient 1 and an overall decrease of all B cell subpopulations, a decreased response to polysaccharide vaccination, and the absence of autoantibodies in patient 2 (Table 2 and Supplementary Table S3). Bone marrow aspiration was once performed in patient 1 , which revealed that $\mathrm{B}$ 


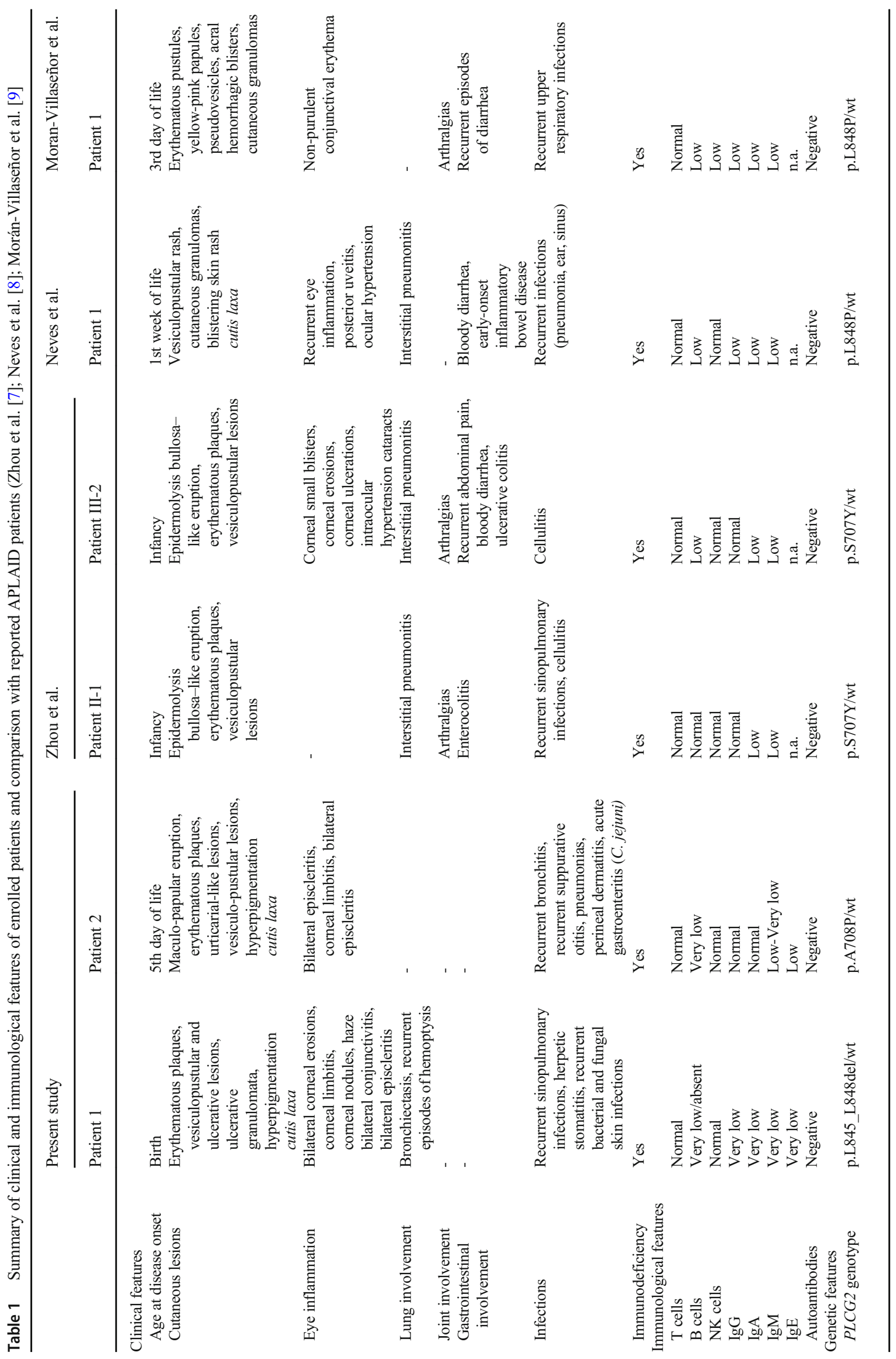


Fig. 2 Description of cutaneous, pulmonary, and ocular inflammatory manifestations in patients. Panel a Multiple papules and serosal and hemorrhagic vesicles on the hands and palms detected in patient 1 at the age of 4 years. Panel b Large areas of cutis laxa in the abdominal region detected in patient 1 at the age of 7 years. Panels c, d Ocular inflammatory lesions including intense bilateral conjunctivitis, keratitis, episcleritis, and nodules in the sclera detected over the course of the disease in patient 1 . Panel e Bronchiectasis detected in patient 1 . Panel $\mathbf{f}$ Blistering inflammatory cutaneous lesions in the leg detected in patient 2 at the age of 6 months. Panel g Areas of cutis laxa detected in patient 2 at the age of 6 years. Panel h Ocular inflammatory lesions including conjunctivitis and corneal limbitis detected in patient 2 at the age of 7 years a

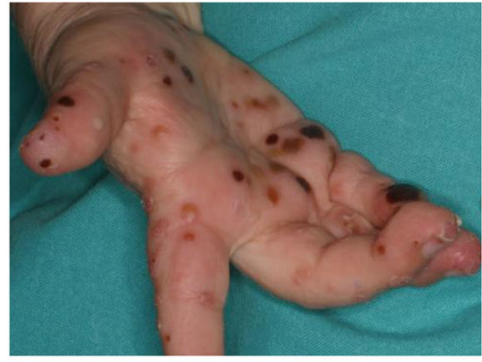

C

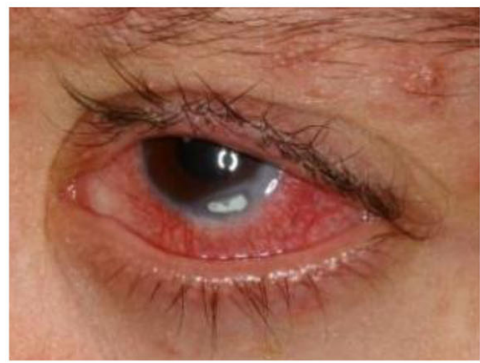

e

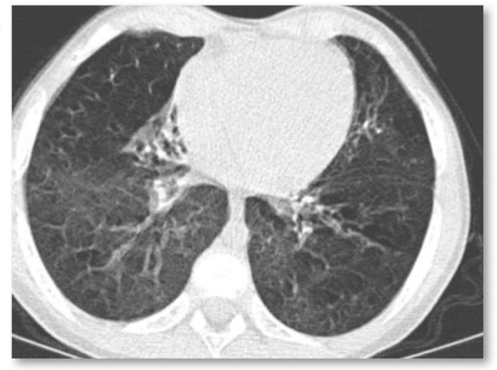

g

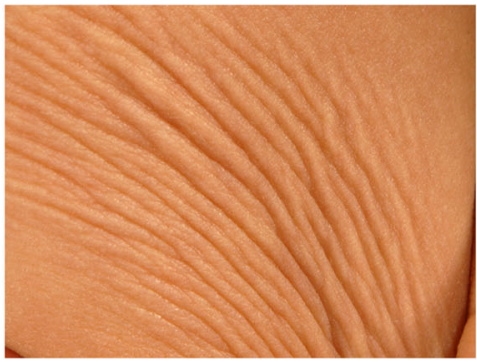

b

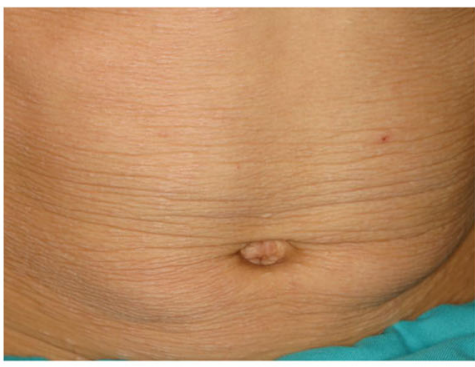

d

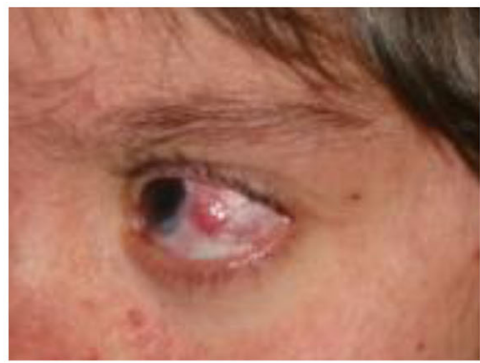

f

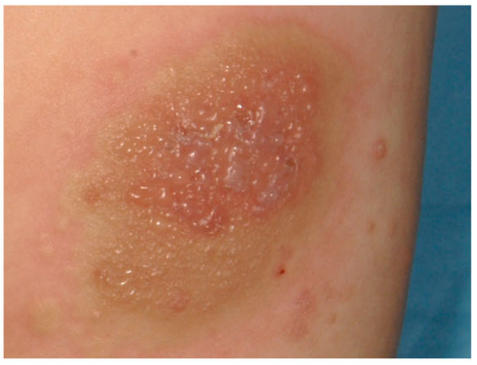

h

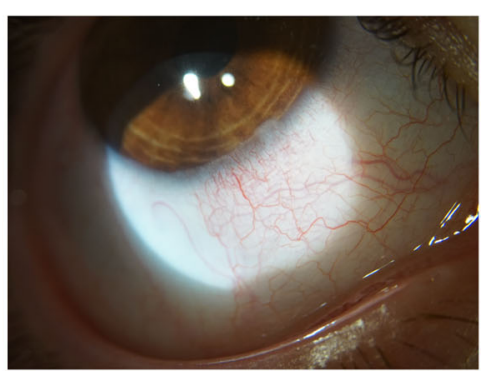

cell lineage represented $6.2 \%$ of total leukocytes and $28.4 \%$ of total lymphocytes, with the presence of all B cell stages. However, a reduction of the immature B cell stage compared with healthy controls was detected (Supplementary Fig. S2).

\section{Molecular Genetics}

Genetic analyses identified rare candidate variants in both patients (Fig. 1b, c shows candidate filtering strategies; Supplementary Table S4 lists the gene variants detected in patient 1). Assuming a dominant inheritance pattern for the disease, patients only shared heterozygous variants at PLCG2 gene (p.Leu845_Leu848del in Family 1 and p.Ala708Pro in Family 2) (Fig. 1d and Table 3). Additional investigations confirmed that they were novel, de novo, and germline
PLCG2 variants (Supplementary Table S5). According to the consensus joint recommendations of the American College of Medical Genetics and Genomics and the Association of Molecular Pathology [14], these two PLCG2 variants were classified as pathogenic on the basis of different criteria including their de novo nature, their absence in healthy controls, their location in regulatory domains and in conserved residues of the protein (Fig. 1e-f), and the results of different bioinformatics and functional analyses (Table 3 ).

\section{Functional Characterization of PLC $\gamma 2$ Variants}

Analyses of $\mathrm{Ca}^{2+}$ flux in $\mathrm{CD} 19^{+}$cells after IgM crosslinking and ionomycin stimulation were performed in both patients using Fluo-3 flow cytometric assay. In patient 1, no firm 


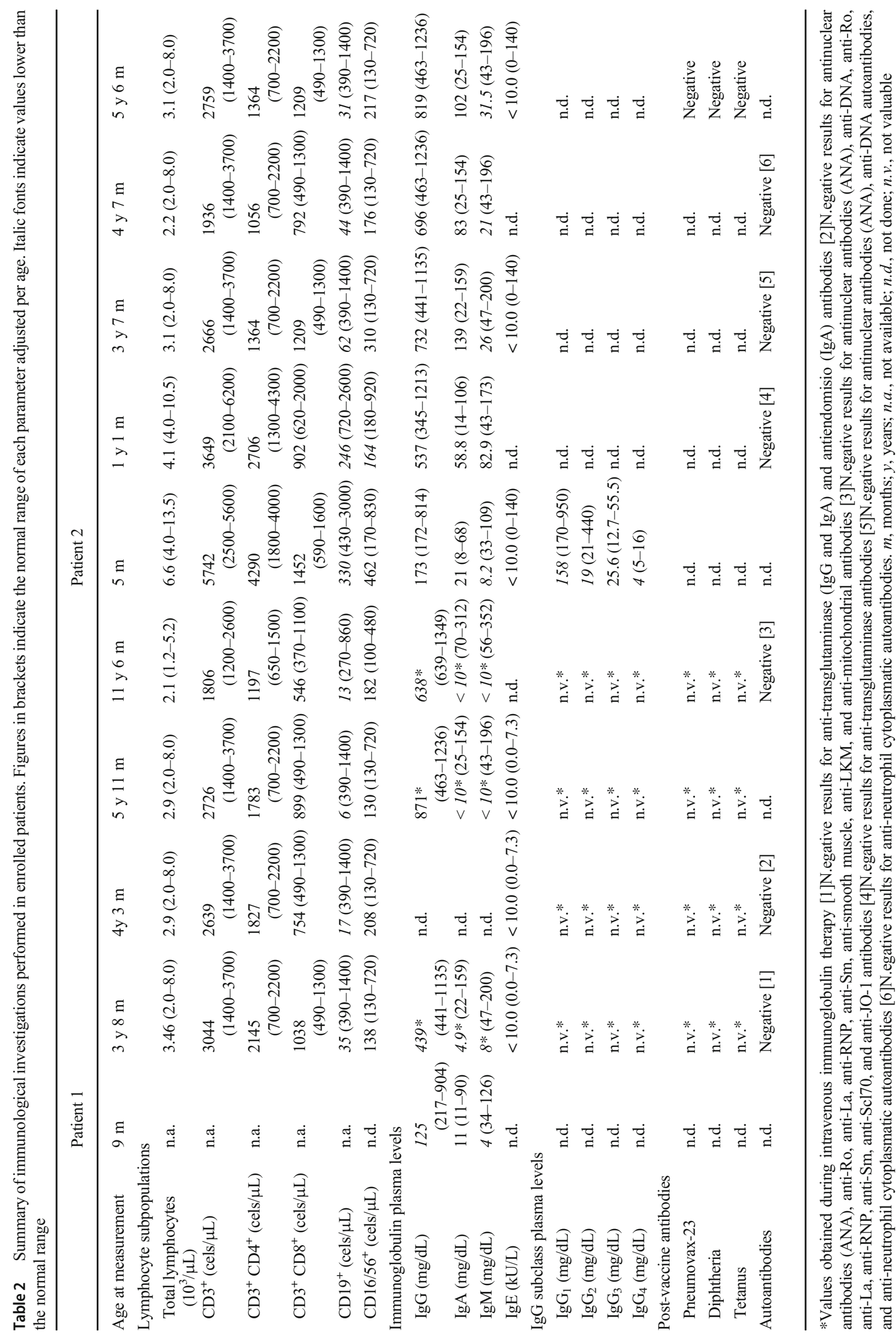


conclusions were drawn from these analyses due to the nearly complete absence of circulating B cells (data not shown). By contrast, analyses performed in patient 2 revealed a significantly higher release of $\mathrm{Ca}^{2+}$ into the cytosol of $\mathrm{B}$ cells after IgM crosslinking stimulation than in cells from control subjects, whereas no significant differences were observed after ionomycin stimulation (Supplementary Fig. 3A-C).

Further analysis of novel APLAID variants was performed in a standard PLC assay, similar to that previously used to measure the activity of PLAID and APLAID variants [6-8]. In this type of analysis, using model cell systems, the substrate is presented in native membranes and the production of inositol phosphates measured under basal or stimulated conditions. As shown in Fig. 3a, both variants have higher PLC activity compared with the wild type with the p.Ala708Pro substitution showing a greater increase in basal and stimulated activities. When analyzed in the context of CLL resistance to ibrutinib, p.Ala708Pro mutation had a pronounced effect [15], consistent with our observations.

\section{NLRP3-Inflammasome Activation and Cytokine Secretion}

PBMCs from APLAID patients showed an increased production of different cytokines after LPS treatment compared with healthy controls, which was similar to that detected in PBMCs from cryopyrin-associated periodic syndrome (CAPS) patients. The upregulated cytokines included proinflammatory cytokines such as TNF- $\alpha$ and different members of IL-1 family (IL-1 $\alpha$, IL-1 $\beta$, IL-1Ra, IL-18) (Fig. 4a). The increased release of IL-1 cytokines occurred simultaneously to an increase of ASC speck formation and activation of caspase-1 on LPS-treated monocytes from APLAID and CAPS patients when compared with monocytes from healthy individuals (Fig. $4 b$ ). IL-1 $\beta$ and IL-18 release from APLAID patients' PBMCs after LPS treatment was comparable to that of CAPS patients (Fig. 4c). The release of IL$1 \beta$ and the activation of caspase- 1 induced by LPS in APLAID PBMCs were reduced when intracellular calcium was chelated with BAPTA-AM or when the widely used PLC inhibitor U73122 was used (Fig. 4d). Canonical activation of the NLRP3-inflammasome by adding ATP or nigericin after LPS priming resulted in an equal formation of intracellular ASC specks in monocytes and similar release of IL-1 $\beta$ in samples from healthy donors, APLAID patients, and CAPS patients (Fig. 4e). Collectively, these results suggest an over-activation of the alternative NLRP3 inflammasome pathway in monocytes from APLAID patients, which could be enhanced by the elevated levels of intracellular calcium associated with the increased activity of mutated PLC $\gamma 2$. 


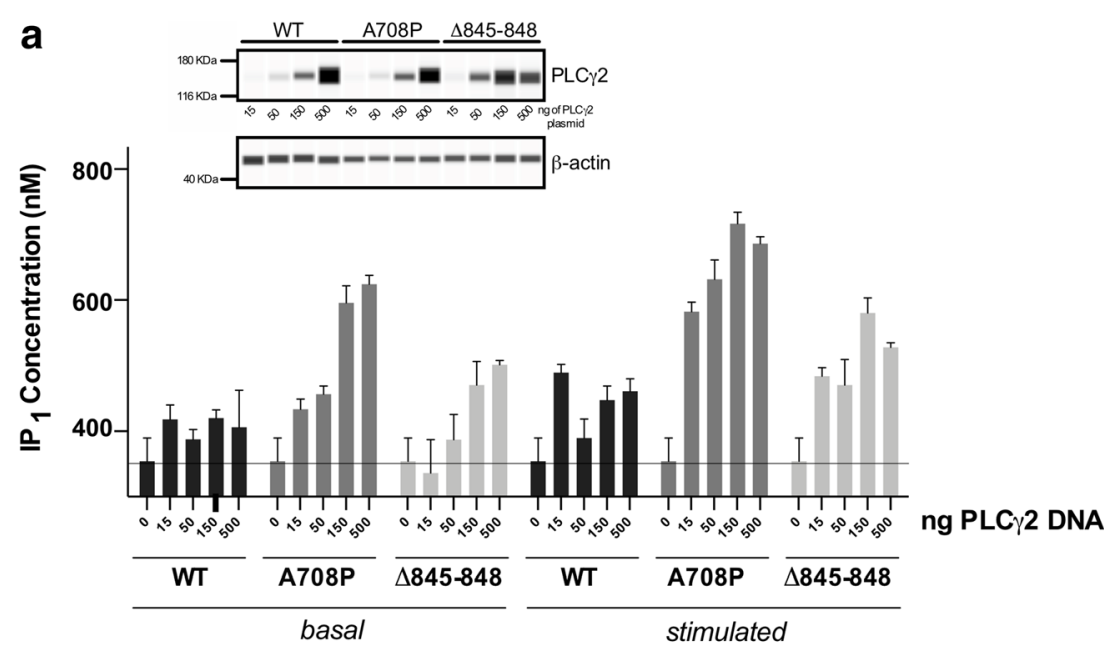

b

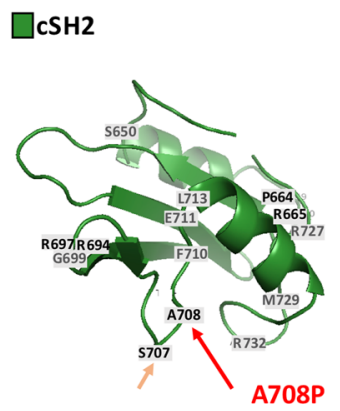

Fig. 3 PLC activity analyses. Panel a The effect of p.Ala708Pro and p.Leu845_Leu848del variants on PLC activity was measured in transfected COS-7 cells under basal conditions (basal) or after stimulation by EGF (stimulated). Each data point represents the mean of triplicates and error bars indicate standard error of the mean. Expression levels of PLC $\gamma 2$, corresponding to increasing concentrations of plasmids used for transfection, were measured using WES (top, inset). Further evaluation of the differences in PLC activities between the WT and variants was performed for the points with an equal protein expression. Panel b

\section{Discussion}

The patients here described were initially suspected of suffering from a rare disease combining humoral immunodeficiency and inflammatory manifestations. Previous genetic studies did not identify defects in different genes causing monogenic antibody deficiencies or AIDs $[1,16]$. Due to strong similarities, we hypothesized that the patients' disease might be a consequence of defects in the same gene. Our further genetic studies revealed that each patient carried a novel, de novo heterozygous PLCG2 pathogenic variant. Furthermore, our extensive characterization of clinical manifestations, properties of immune cells, and changes in function of the encoded enzyme, PLC $\gamma 2$, support a definitive diagnosis of PLC $\gamma 2$-associated antibody deficiency and immune dysregulation syndrome, designated as APLAID.

Previously, only 4 patients from three families have been diagnosed with APLAID [7-9] and have been found to share some of the manifestations with Ali5 and Ali14 mouse strains

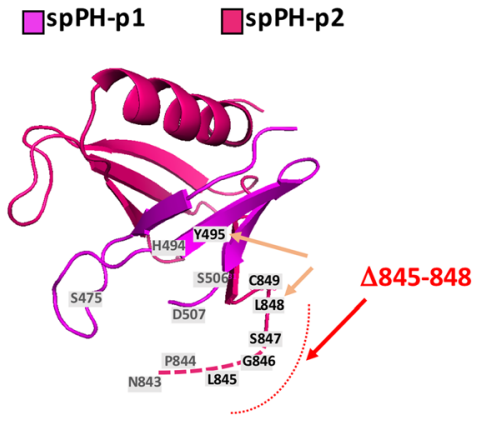

Position of p.Ala708 and p.Leu845-Leu848 segment (red arrows) is mapped on the structure of $\mathrm{cSH} 2$ and $\mathrm{spPH}$ domain, respectively. Positions of other mutations, reported for the main PLC $\gamma 1$ - and PLC $\gamma 2$ linked pathologies that map to the same domains, are labeled using single letters and numbers corresponding to PLC $\gamma 2$ sequence. Residues so far found to be mutated only in PLC $\gamma 1$ are shown in gray. Other residues mutated in APLAID (p.Ser707 and p.Leu848) and Ali14 mice (p.Tyr495) are indicated by orange arrows

that carry gain-of-function PLCG2 mutations [17, 18]. Another two families carrying the same PLCG2 variant in the $\mathrm{C} 2$ domain of the protein have been recently described during manuscript reviewing [19]. The two unrelated patients described in our study enable us to make more extensive comparisons and highlight the key changes that characterize the human syndrome and parallels with the mouse models. These include severe inflammatory lesions at skin, ocular, and joints mediated by non-lymphoid hematopoietic cells and variable degree of immunodeficiency. With regard to the immune defects, all known APLAID patients exhibited variable degrees of B cell lymphopenia and antibody deficiency, with no apparent impairment of $\mathrm{T}$ and NK functions (Table 1). Patient 1 carrying the p.Leu845 Leu848 PLCG2 deletion showed the complete absence of B cells and circulating immunoglobulins, an immune phenotype compatible with a rare form of non-X-linked agammaglobulinemia [16] and quite similar to the immune phenotype of the APLAID patients carrying the missense 


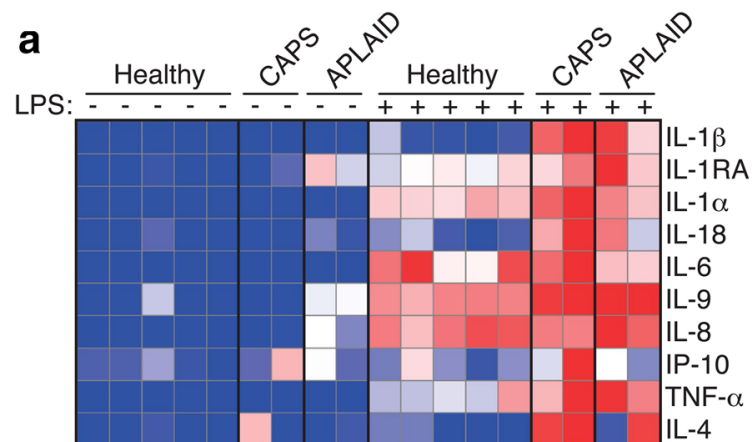

\section{b}

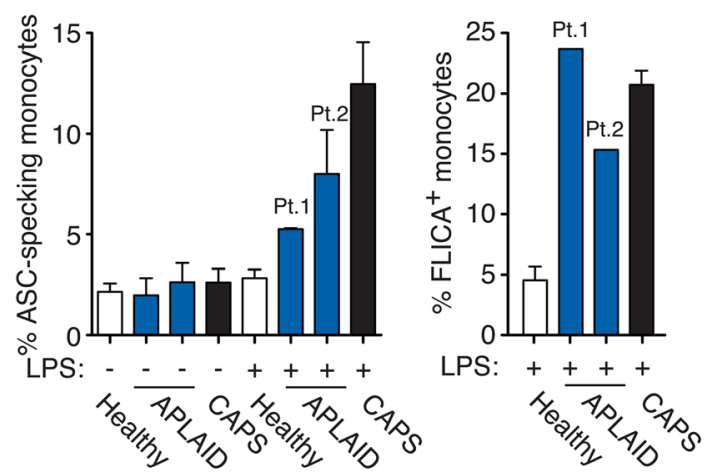

C
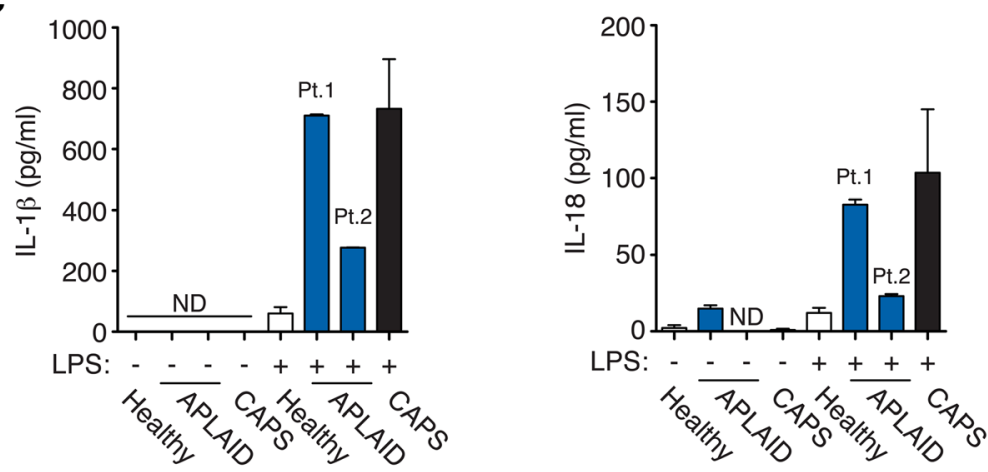

d
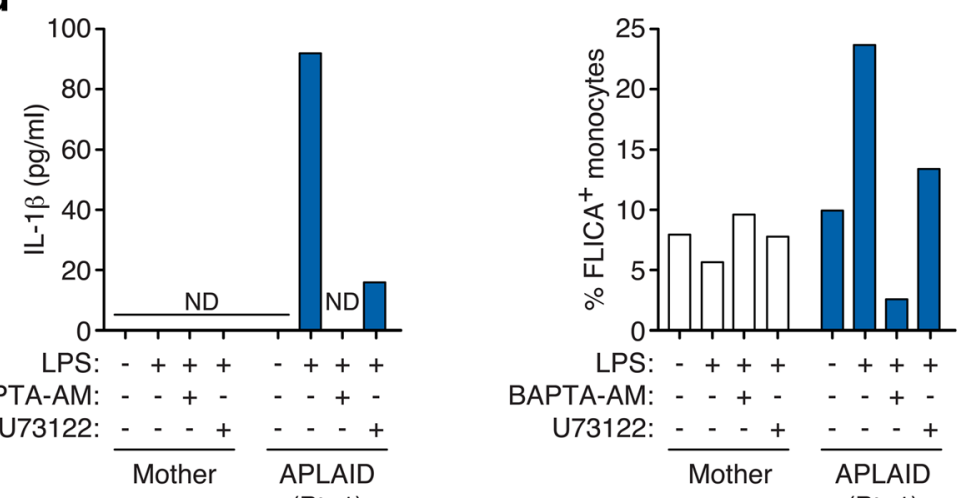

BAPTA-AM: - - + - - +

U73122: $\frac{--++}{\text { Mother }} \frac{--+}{\text { APLAID }}$

(Pt. 1)

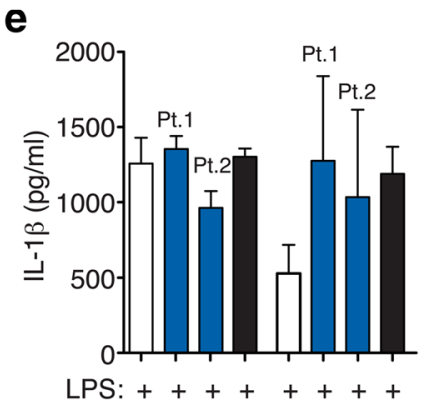

LPS: ++++++++

ATP: ++++---
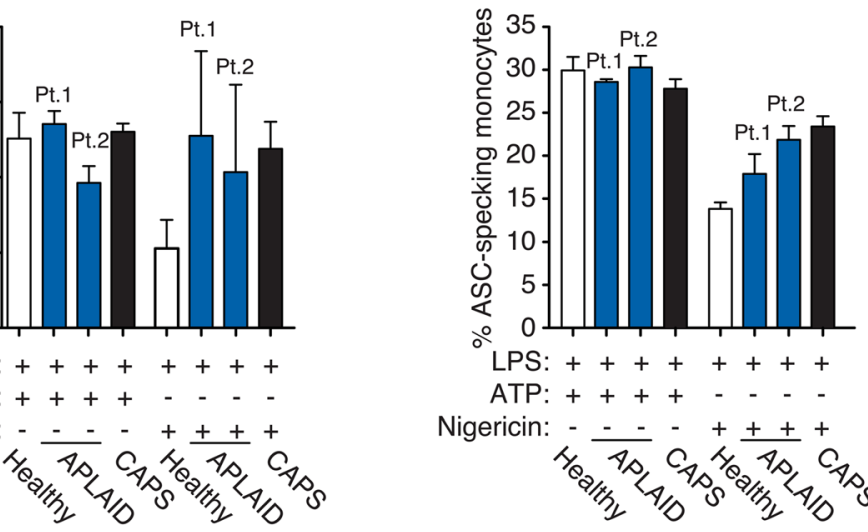

LPS: ++++++++
ATP: $++++-{ }_{-}+$

Nigericin:

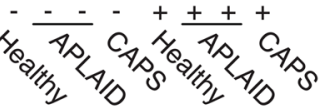


Fig. 4 Involvement of NLRP3-inflammasome activation in sterile inflammation. Panel a Heat map of cytokine analysis from peripheral blood mononuclear cell (PBMCs) supernatants after LPS stimulation as indicated $(1 \mu \mathrm{g} / \mathrm{mL}, 2 \mathrm{~h})$ isolated from healthy controls $(n=5)$, patients with CAPS (heterozygous for p.Arg260Trp NLRP3 mutation; $n=2$ ) or APLAID carrying the heterozygous p.A708P $(n=1)$ or p.Leu845 Leu848del $(n=1)$ PLCG2 variants. Representative of relative values of minimum and maximum concentrations measured per cytokine. Panel b Apoptosis-associated Speck-like protein containing a Caspase recruitment domain (ASC) speck forming monocytes by flow cytometry and active caspase- 1 by YVAD-Fluorochrome Inhibitor of Caspases (FLICA) staining on monocytes after LPS stimulation as indicated $(1 \mu \mathrm{g} / \mathrm{mL}, 2 \mathrm{~h})$ isolated from healthy controls, patients with CAPS, or patients with APLAID. Showed results are representative of duplicate experiments. Panel c PBMCs IL-1 $\beta$ and IL-18 cytokine production at baseline and after LPS stimulation as indicated $(1 \mu \mathrm{g} / \mathrm{mL}$, $2 \mathrm{~h}$ ) in healthy controls, patients with CAPS, and patients with APLAID. Showed results are representative of duplicate experiments. Panel d PBMCs IL- $1 \beta$ and percentage of monocytes stained for active caspase1 by FLICA at baseline and after LPS stimulation $(1 \mu \mathrm{g} / \mathrm{mL}, 2 \mathrm{~h})$ in the presence or absence of BAPTA-AM $(20 \mu \mathrm{M})$ or U73122 $(2.5 \mu \mathrm{M})$ as indicated in APLAID patient 1 (p.Leu845_Leu848del PLCG2 variant) and her healthy mother. Showed results are representative of experiments performed only once due to limited availability of samples. Panel e PBMCs IL-1 $\beta$ and ASC speck forming monocytes upon canonical NLRP3 activation by LPS priming $(1 \mu \mathrm{g} / \mathrm{mL}, 2 \mathrm{~h})$ followed by $30-\mathrm{min}$ treatment with ATP $(3 \mathrm{mM})$ or nigericin $(10 \mu \mathrm{M})$ in healthy controls, patients with CAPS, and patients with APLAID. Showed results are representative of duplicate experiments. ND, not detected

p.Leu848Pro PLCG2 variant $[8,9]$. The humoral immunodeficiency of this patient (patient 1 ) was clinically characterized by severe and recurrent bacterial infections, structural lung lesions, recurrent episodes of acute hemoptysis, and the requirement of surgical lung lobe resection. To our knowledge, this patient represents the severest APLAID patient described to date and clearly expands the diversity of the immune deficiency of this disease toward phenotypes compatible with dominantly inherited agammaglobulinemia. Since allogeneic hematopoietic stem cell transplantation has been proposed as a curative option in severe PID [20, 21], a major unsolved question is whether this particular patient would have benefited from this therapeutic approach at a younger age, even with the causative genetic defect not elucidated. With regard to patient 2 who carried the missense p.Ala708Pro PLCG2 variant, his immunological phenotype (low IgM and B cell count and decreased circulating class-switched memory B cells) could be classified as mild-tomoderate and displayed marked similarities with the phenotype of the first described APLAID family carrying the missense p.Ser707Tyr PLCG2 variant [7]. The B cell disturbances observed in patients with APLAID are variable ranging from patients displaying low circulating B cells to patients with absent B cells. PLCG2 was proposed as a good candidate gene to explain some $B$ cell deficiencies in humans because the mouse model of PLC $\gamma 2$ deficiency showed a decrease of mature B cell and a block in the pro-B cell differentiation [22]. However, the human deficiency of PLC $\gamma 2$ has not been yet described. By contrast, monoallelic $P L C G 2$ mutations are associated with variable humoral immune deficiency and immune dysregulation in PLAID and APLAID syndromes [6-9]. The few available data about the defects of the B cell lineage in APLAID patients do not permit to drawn firm conclusions about the precise molecular mechanisms underlying the humoral deficiency in these patients. Considering the relevant role of PLC $\gamma 2$ in multiple signal transduction pathways in B cells and the gain-of-function nature of PLCG2 variants detected in APLAID syndrome, a mechanism that may explain the immune phenotype observed and that should be further investigated is the deletion and/or functional anergy of specific B cell progenitors due to enhanced cellular signaling.

We were particularly interested in sterile inflammatory lesions and their molecular basis. From a clinical perspective, the cutaneous manifestations were the most prominent in both of our patients (patient 1 and 2) since the onset of their diseases as have been also described for the other four published APLAID patients [7-9]. These manifestations were initially vesicular and blistering lesions that subsequently evolved to the destruction of elastin fibers leading to large areas of cutis laxa in both patients, a phenomenon also described in the APLAID patient with the p.Leu848Pro PLCG2 variant [8]. Later in life both patients also developed severe eye inflammation, mainly affecting conjunctiva and episclera. The beneficial effect of topic anakinra in the ocular lesions in PLAID [23] suggests the potential combination of topical ocular treatments to the systemic therapies that both patients are already receiving.

We have further investigated the molecular mechanisms underlying sterile inflammation in APLAID. We found that the novel PLC $\gamma 2$ variants here identified were able to induce activation of the NLRP3 inflammasome, with ASC aggregation, caspase- 1 activation, and IL-1 $\beta$ and IL-18 release in monocytes. Previous evidence suggested a potential connection between hypermorphic PLCG2 mutations and NLRP3inflammasome activation throughout the increase of intracellular calcium [7, 16, 24, 25]. Consequently, it is possible that in the patients described here, the sterile inflammation manifestations could be also linked to NLRP3-inflammasome. Our results showed that canonical activation pathway of NLRP3inflammasome in patient's monocytes did not result in an increase of IL-1 $\beta$ release or ASC-specking as observed for healthy monocytes. Therefore, the inflammasome pathway that is potentiated in APLAID might be related to the alternative NLRP3 activation pathway, similar to that described in human monocytes upon LPS activation [26], rather than the canonical pathway that requires ATP or nigericin as second stimuli after LPS priming [27].

Finally, our identification of two new pathogenic variants in PLCG 2 linked to APLAID (p.Ala 708 Pro and p.Leu845 Leu848) expands the range of genetic changes that cause this syndrome. Consistent with previous studies [7-9], we show that the new PLCG2 variants have higher PLC 
activity compared with the wild type. Interestingly, p.Ala708Pro PLCG2 variant has been also discovered in ibrutinib-resistant chronic lymphocytic leukemia (CLL) in the form of somatic variant [28-30]. Two other somatic PLCG2 variants detected in resistant CLL, p.Ser707Tyr and p.Asp993Gly, are also shared with immune disorders in APLAID [7] and Ali5 mice [17], respectively. More broadly, the new variants map to distinct regions in the $\mathrm{cSH} 2$ domain, and $\mathrm{spPH}$ domain and its vicinity, the regions that appear to harbor mutations across different PLC $\gamma$-linked pathologies with higher frequencies (Fig. 3b). Therefore, the molecular mechanisms that lead to an increase in PLC activity in diverse pathologies could be similar. Considerable supporting evidence is consistent with a model where PLC $\gamma 2$ is kept in an inactive state by extensive intramolecular interactions between the regulatory domains (including $\mathrm{cSH} 2$ and $\mathrm{spPH}$ domains) and the PLC-core domains [11, 31]. Release of this autoinhibition by physiological stimulation (via phosphorylation) or by mutations represents an important step leading to an increase in PLC activity. Therefore, our data showing that the novel variants are hypermorphic and their position in the protein highlight particular structural features within the regulatory array that are the key regions involved in autoinhibition.

In conclusion, from the perspective of clinical practice, the detection of a novel, de novo hypermorphic PLCG2 pathogenic variant in each of the two patients displaying early-onset severe skin and eye inflammation and humoral immunodeficiency supports their definitive diagnosis of the extremely rare APLAID syndrome. Moreover, the patients' immunological features expand the APLAID diversity toward most severe phenotypes than previously described including complete B cell depletion and agammaglobulinemia inherited as a dominant trait, which should be considered when evaluating patients with severe deficiencies of antibody production.

Acknowledgments We would like to particularly acknowledge the patients and their families for their collaboration in this study. We would also like to acknowledge the Biobank of Vall d'Hebron Research Institute (PT13/0010/0021), integrated in the Spanish National Biobanks Network, and its director Isabel Novoa for her help in this study. The authors would like to acknowledge Mr. William Sinclair for his help in language editing.

Authorship Contributions JIA, PP, and MK designed and wrote the manuscript. AM-N, CF, LR, MG-P, NM-B, VG-P, RMP, PS-P, and $\mathrm{AV}$ collected clinical data and provided samples of patients and relatives for genetic and molecular analyses. FR, RC, FC, RR, and XE performed and analyzed the results of next-generation sequencing. MCA and JA performed and analyzed Sanger sequencing in both families. LA, AD, RG, AM-V, MJ, JY, and JIA performed and analyzed immunological tests of patients. MB provided samples from patients with cryopyrinassociated periodic syndromes. HM-B, JJM-G, and PP performed analyses of inflammasome activation and cytokine quantification. AE-S performed ex vivo $\mathrm{Ca}^{+2}$ flux assays. TDB, DB, and MK evaluated in vitro PLC activity of mutant alleles. All authors reviewed, contributed, and approved the final draft of the manuscript.
Funding Information This work has been partially funded by the following: CERCA Programme/Generalitat de Catalunya (JIA), SAF201568472-C2-1-R grant from the Spanish Ministry of Economy and Competitiveness co-financed by European Regional Development Fund (ERDF) (JIA), RTI2018-096824-B-C21 grant from the Spanish Ministry of Science, Innovation and Universities co-financed by ERDF (JIA), AC15/00027 grant from the Instituto de Salud Carlos III/Transnational Research Projects on Rare Diseases (JA), PI14/00405 grant from the Instituto de Salud Carlos III co-financed by ERDF (RC), PI13/00174 grant from the Spanish Ministry of Economy and Competitiveness cofinanced by ERDF (PP), SAF2017-88276-R grant from the Spanish Ministry of Economy, Industry and Competitiveness co-financed by ERDF (PP), ERC-2013-CoG project 614578 from the European Research Council (PP), 20859/PI/18 grant from Fundación Séneca (PP), SAF2015-68472-C2-2-R grant from the Spanish Ministry of Economy and Competitiveness co-financed by ERDF (FC) and RTI2018-096824B-C22 grant from the Spanish Ministry of Science, Innovation and Universities co-financed by ERDF (FC). MK acknowledges support from CRUK (A16567) and MRC (P028160). H M-B is a Rio Hortega fellowship from Instituto de Salud Carlos III (CM14/00008) and DB acknowledges support from the UCL Impact Studentship.

\section{Compliance with Ethical Standards}

The ethics committees of Hospital Sant Joan de Déu, Hospital Universitari Vall d'Hebron, and Hospital Clínic, all in Barcelona (Spain), approved the study. Written informed consent for participation in the study was obtained from patients' parents. Blood samples from patients and unaffected relatives were collected for molecular studies, which were performed in accordance with the Declaration of Helsinki.

Conflict of Interest The authors declare that they have no conflict of interest.

Open Access This article is licensed under a Creative Commons Attribution 4.0 International License, which permits use, sharing, adaptation, distribution and reproduction in any medium or format, as long as you give appropriate credit to the original author(s) and the source, provide a link to the Creative Commons licence, and indicate if changes were made. The images or other third party material in this article are included in the article's Creative Commons licence, unless indicated otherwise in a credit line to the material. If material is not included in the article's Creative Commons licence and your intended use is not permitted by statutory regulation or exceeds the permitted use, you will need to obtain permission directly from the copyright holder. To view a copy of this licence, visit http://creativecommons.org/licenses/by/4.0/.

\section{References}

1. Manthiram K, Zhou Q, Aksentijevich I, Kastner DL. The monogenic autoinflammatory diseases define new pathways in human innate immunity and inflammation. Nat Immunol. 2017;18:83242.

2. Liu Y, Jesus AA, Marrero B, Yang D, Ramsey SE, Montealegre Sanchez GA, et al. Activated STING in a vascular and pulmonary syndrome. N Engl J Med. 2014;371:507-18.

3. Zhou Q, Yang D, Ombrello AK, Zavialov AV, Toro C, Zavialov $\mathrm{AV}$, et al. Early-onset stroke and vasculopathy associated with mutations in ADA2. N Engl J Med. 2014;370:911-20. 
4. Navon Elkan P, Pierce SB, Segel R, Walsh T, Barash J, Padeh S, et al. Mutant adenosine deaminase 2 in a polyarteritis nodosa vasculopathy. N Engl J Med. 2014;370:921-31.

5. Chakraborty PK, Schmitz-Abe K, Kennedy EK, Mamady H, Naas $\mathrm{T}$, Durie D, et al. Mutations in TRNT1 cause congenital sideroblastic anemia with immunodeficiency, fevers, and developmental delay (SIFD). Blood. 2014;124:2867-71.

6. Ombrello MJ, Remmers EF, Sun G, Freeman AF, Datta S, TorabiParizi P, et al. Cold urticaria, immunodeficiency and autoimmunity related to PLCG2 deletions. N Engl J Med. 2012;366:330-8.

7. Zhou Q, Lee G-S, Brady J, Datta S, Katan M, Sheikh A, et al. A hypermorphic missense mutation in PLCG2, encoding phospholipase $\mathrm{C} \gamma 2$, causes a dominantly-inherited autoinflammatory disease with immunodeficiency. Am J Hum Genet. 2012;91:713-20.

8. Neves JF, Doffinger R, Barcena-Morales G, Martins C, Papapietro $\mathrm{O}$, Plagnol V, et al. Novel PLCG2 mutation in a patient with APLAID and cutis laxa. Front Immunol. 2018;9:2863.

9. Morán-Villaseñor E, Saez-de-Ocariz M, Torrelo A, Arostegui JI, Yamazaki-Nakashimada MA, Alcántara-Ortigoza MA, et al. Expanding the clinical features of autoinflammation and PLC $\gamma 2$ associated antibody deficiency and immune dysregulation by description of a novel patient. J Eur Acad Dermatol Venereol. 2019;33:2334-9.

10. Mensa-Vilaró A, Bravo García-Morato M, de la Calle-Martin O, Franco-Jarava C, Martínez-Saavedra MT, González-Granado LI, et al. Unexpected relevant role of gene mosaicism in patients with primary immunodeficiency diseases. J Allergy Clin Immunol. 2019; 143:359-68.

11. Liu Y, Bunney TD, Khosa S, Macé K, Beckenbauer K, Askwith T, et al. Structural insights and activating mutations in diverse pathologies define mechanisms of deregulation for phospholipase $\mathrm{C}$ gamma enzymes. EBioMedicine. 2020;51:102607.

12. Sester DP, Thygesen SJ, Sagulenko V, Vajjhala PR, Cridland JA, Vitak N, et al. A novel flow cytometry method to assess inflammasome formation. J Immunol. 2015;194:455-62.

13. Torrelo A, Vera A, Portugués M, de Prada I, Sanz A, Colmenero I, et al. Perforating neutrophilic and granulomatous dermatitis of the newborn: a clue to immunodeficiency. Pediatr Dermatol. 2007;24: 211-5.

14. Richards S, Aziz N, Bale S, Bick D, Das S, Gastier-Foster J, et al. Standards and guidelines for the interpretation of sequence variants: a joint consensus recommendation of the American College of Medical Genetics and Genomics and the Association for Molecular Pathology. Genet Med. 2015;17:405-24.

15. Walliser C, Wist M, Hermkes E, Zhou Y, Schade A, Haas J, et al. Functional characterization of phospholipase $\mathrm{C}-\gamma(2)$ mutant protein causing both somatic ibrutinib resistance and a germline monogenic autoinflammatory disorder. Oncotarget. 2018;9:34357-78.

16. Berglöf A, Turunen JJ, Gissberg O, Bestas B, Blomberg KE, Smith CI. Agammaglobulinemia: causative mutations and their implications for novel therapies. Expert Rev Clin Immunol. 2013;9:120521.

17. Yu P, Constien R, Dear N, Katan M, Hanke P, Bunney TD, et al. Autoimmunity and inflammation due to a gain-of-function mutation in phospholipase $\mathrm{C}$ gamma 2 that specifically increases external Ca2+ entry. Immunity. 2005;22:451-65.
18. Abe K, Fuchs H, Boersma A, Hans W, Yu P, Kalaydjiev S, et al. A novel $\mathrm{N}$-ethyl-N-nitrosourea-induced mutation in phospholipase $\mathrm{C} \gamma 2$ causes inflammatory arthritis, metabolic defects, and male infertility in vitro in a murine model. Arthritis Rheum. 2011;63: 1301-11.

19. Novice T, Kariminia A, Del Bel KL, Lu H, Sharma M, Lim CJ, et al. A germline mutation in the $\mathrm{C} 2$ domain of PLC $\gamma 2$ associated with gain-of-function expands the phenotype for PLCG2-related diseases. J Clin Immunol. 2020;40:267-76.

20. Griffith LM, Cowan MJ, Notarangelo LD, Kohn DB, Puck JM, Shearer WT, et al. Primary immune deficiency treatment consortium (PIDTC) update. J Allergy Clin Immunol. 2016;138(2):37585 .

21. Wehr C, Gennery AR, Lindemans C, Schulz A, Hoenig M, Marks $\mathrm{R}$, et al. Multicenter experience in hematopoietic stem cell transplantation for serious complications of common variable immunodeficiency. J Allergy Clin Immunol. 2015;135(4):988-97.

22. Wang D, Feng J, Wen R, Marine J-C, Sangster MY, Parganas E, et al. Phospholipase $\mathrm{C} \gamma 2$ is essential in the functions of $\mathrm{B}$ cell and several Fc receptors. Immunity. 2000;13:25-35.

23. Di Zazzo A, Tahvildari M, Florakis GJ, Dana R. Ocular manifestations of inherited phospholipase- $\mathrm{C} \gamma 2$-associated antibody deficiency and immune dysregulation. Cornea. 2016;35:1656-7.

24. Lee GS, Subramanian N, Kim AI, Aksentijevich I, GoldbachMansky R, Sacks DB, et al. The calcium-sensing receptor regulates the NLRP3 inflammasome through $\mathrm{Ca} 2+$ and cAMP. Nature. 2012;492:123-7.

25. Chae JJ, Park YH, Park C, Hwang I, Hoffmann P, Kehrl JH, et al. Connecting two pathways through $\mathrm{Ca} 2+$ signaling: NLRP3 Inflammasome activation induced by a hypermorphic PLCG2 mutation. Arthritis Rheum. 2015;67:563-7.

26. Gaidt MM, Ebert TS, Chauhan D, Schmidt T, Schmid-Burgk JL, Rapino F, et al. Human monocytes engage an alternative Inflammasome pathway. Immunity. 2016;44:833-46.

27. de Torre-Minguela C, Mesa Del Castillo P, Pelegrín P. The NLRP3 and pyrin inflammasomes: implications in the pathophysiology of autoinflammatory diseases. Front Immunol. 2017;8:43.

28. Woyach JA, Furman RR, Liu TM, Ozer HG, Zapatka M, Ruppert AS, et al. Resistance mechanisms for the Bruton's tyrosine kinase inhibitor ibrutinib. N Engl J Med. 2014;370:2286-94.

29. Liu TM, Woyach JA, Zhong Y, Lozanski A, Lozanski G, Dong S, et al. Hypermorphic mutation of phospholipase $\mathrm{C} \gamma 2$ acquired in ibrutinib-resistant CLL confers BTK independency upon B-cell receptor activation. Blood. 2015;126(1):61-8.

30. Koss H, Bunney TD, Behjati S, Katan M. Dysfunction of phospholipase $\mathrm{C} \gamma$ in immune disorders and cancer. Trends Biochem Sci. 2014;39:603-11.

31. Hajicek N, Keith NC, Siraliev-Perez E, Temple BR, Huang W, Zhang Q, et al. Structural basis for the activation of PLC- $\gamma$ isozymes by phosphorylation and cancer-associated mutations. Elife. 2019;8:e51700.

Publisher's Note Springer Nature remains neutral with regard to jurisdictional claims in published maps and institutional affiliations. 


\section{Affiliations}

Andrea Martín-Nalda ${ }^{1,2}$. Claudia Fortuny ${ }^{3,4,1}$ • Lourdes Rey ${ }^{5} \cdot$ Tom D. Bunney ${ }^{6}$ - Laia Alsina ${ }^{4,7,8}$ • Ana Esteve-Solé ${ }^{4,7,8}$. Daniel Bull $^{9}$ - Maria Carmen Anton ${ }^{10}$ - María Basagaña ${ }^{11}$ • Ferran Casals ${ }^{12}$ • Angela Deyá ${ }^{4,7,8}$ - Marina García-Prat ${ }^{1,2}$. Ramon Gimeno ${ }^{13}$. Manel Juan ${ }^{10,14,15}$ • Helios Martinez-Banaclocha ${ }^{16}$ • Juan J Martinez-Garcia ${ }^{16}$. $^{1}$ Anna Mensa-Vilaró ${ }^{10} \cdot$ Raquel Rabionet $^{4,17}$ • Nieves Martin-Begue ${ }^{18}$. Francesc Rudilla ${ }^{19,20} \cdot$ Jordi Yagüe $^{10,14,15}$. Xavier Estivill ${ }^{21}$ - Vicente García-Patos ${ }^{22}$ - Ramon M. Pujol ${ }^{23}$ - Pere Soler-Palacín ${ }^{1,2,24}$ • Matilda Katan ${ }^{6}$. Pablo Pelegrín ${ }^{16}$ • Roger Colobran ${ }^{2,25,26}$ • Asun Vicente ${ }^{27} \cdot$ Juan I. Arostegui ${ }^{10,14,15}$

1 Pediatric Infectious Diseases and Immunodeficiencies Unit, Vall d'Hebron Institut de Recerca, Hospital Universitari Vall d'Hebron, Barcelona, Spain

2 Jeffrey Modell Diagnostic and Research Center for Primary Immunodeficiencies, Barcelona, Spain

3 Department of Pediatrics, Hospital Sant Joan de Deu, Esplugues, Spain

4 Institut de Recerca Hospital Sant Joan de Déu, Universitat de Barcelona, Esplugues, Spain

5 Department of Pediatrics, Hospital Alvaro Cunqueiro, Vigo, Spain

6 Institute of Structural and Molecular Biology, University College London, London, UK

7 Department of Allergy and Clinical Immunology Clinical Immunology and Primary, Immunodeficiencies Unit, Hospital Sant Joan de Déu, Esplugues, Spain

8 Clinical Immunology Unit, Hospital Sant Joan de Déu-Hospital Clínic, Barcelona, Spain

9 ARUK Drug Discovery Institute, University College London, London, UK

10 Department of Immunology-CDB (esc 4-pl 0), Hospital Clínic, Villarroel, 170, 08036 Barcelona, Spain

11 Allergy Section, Hospital Universitari Germans Trias i Pujol, Autonomous University of Barcelona, Badalona, Spain

12 Genomics Core Facility, Experimental and Health Sciences Department, Universitat Pompeu Fabra, Barcelona, Spain

13 Department of Immunology, Hospital del Mar, Institut Mar d'Investigacions Mèdiques, Barcelona, Spain

14 Institut d'Investigacions Biomèdiques August Pi i Sunyer, Barcelona, Spain
15 School of Medicine, Universitat de Barcelona, Barcelona, Spain

16 Instituto Murciano de Investigación Biosanitaria IMIB-Arrixaca, Hospital Clínico Universitario Virgen de la Arrixaca, Murcia, Spain

17 Department of Genetics, Microbiology and Statistics, Faculty of Biology, University of Barcelona, IBUB, IRJSD, CIBERER, Barcelona, Spain

18 Department of Pediatric Ophthalmology, Hospital Universitari Vall d'Hebron, Vall d'Hebron Institut de Recerca, Barcelona, Spain

19 Histocompatibility and Immunogenetics Laboratory, Blood and Tissue Bank, Barcelona, Spain

20 Transfusional Medicine Group, Vall d'Hebron Research Institute, Autonomous University of Barcelona, Barcelona, Spain

21 Quantitative Genomic Medicine Laboratories (qGenomics), Esplugues del Llobregat, Barcelona, Catalonia, Spain

22 Department of Pediatric Dermatology, Hospital Universitari Vall d'Hebron, Vall d'Hebron Institut de Recerca, Barcelona, Spain

23 Department of Dermatology, Hospital del Mar, Institut Mar d'Investigacions Mèdiques, Universitat Autonoma de Barcelona, Barcelona, Spain

24 Universitat Autonoma de Barcelona, Barcelona, Spain

25 Immunology Division, Department of Clinical and Molecular Genetics, Hospital Universitari Vall d'Hebron, Vall d'Hebron Research Institute, Barcelona, Spain

26 Department of Cell Biology, Physiology and Immunology, Autonomous University of Barcelona, Barcelona, Spain

27 Department of Pediatric Dermatology, Hospital Sant Joan de Deu, Esplugues, Spain 